\section{The Original Source of Modern Research on Chinese Medicinal Materials: Bencao Texts Zhitao Liang and Zhongzhen Zhao*}

School of Chinese Medicine, Hong Kong Baptist University, Kowloon Tong, Hong Kong

\begin{abstract}
Bencao texts, the unique name of the Chinese traditional materia medica, record the knowledge of Chinese medicinal materials. Numerous Bencao texts have been circulated since the end of warring states period. The paper outlines the contents and modern research of Bencao texts. Also the impact of Bencao texts on modern research of Chinese Medicinal Materials (CMMs) is described. Briefly, Bencao texts can be mainly classified as main Bencao texts in the past dynasties, theme of Bencao texts and local Bencao texts. Much research work on Bencao texts has been carried out on the literature and textual study. Many lost Bencao texts have been reconstructed by later generations of editors and the contents of extant Bencao texts have been well studied. Inspired from Bencao texts, the development of new drugs and resources, as well as the modern quality evaluation and safety of CMMs has achieved many results. The purpose of current paper is to arouse the attention to Bencao texts and the intellectual property rights protection of the traditional knowledge recorded in Bencao texts.
\end{abstract}

Keywords: Bencao text; Chinese Medicinal Materials; Intellectual property rights; Textual study

\section{Introduction}

According to ancient Chinese tradition, the mythological founder of Chinese herbal medicine, Shen Nong (the "Divine Husbandman"), was said to have "tried the hundred herbs by tasting them, encountering 70 toxicities within a day". This description of Shen Nong was recorded in the early Western Han Dynasty in the ancient text, the

*Corresponding author: Zhongzhen Zhao, School of Chinese Medicine, Hong Kong Baptist University, Kowloon Tong, Hong Kong, Tel: +852 34112424; E-mail: zzzhao@hkbu.edu.hk

Citation: Zhao Z, Liang Z (2017) The Original Source of Modern Research on Chinese Medicinal Materials: Bencao Texts. J Altern Complement Integr Med 3: 045.

Received: November 07, 2017; Accepted: December 25, 2017; Published: December 29, 2017
Philosophers of Huainan (Huai Nan Zi, ca. 200 B.C.E.). Valuable experiences accumulated through ancient human trial and error have been passed down from generation to generation in a rich oral and literary history, and the myth of Shen Nong honors the countless unknown individuals that dedicated their lives to discovering the effects of the botanicals around them.

The broad genre of Chinese materia medica texts is often referred to as "Bencao" literature. This term, which literally means "rooted in herbs," implies that medicinal materials are primarily plant-derived. These various Bencao texts (materia medica) in documenting ancient people's experience represents centuries of accumulated wisdom in combating disease and preserving health. Bencao texts primarily consist of records related to three different categories: the medicinal material itself, its medicinal effects, and its medicinal nature. In discussions about the medicinal material itself, Bencao texts primarily emphasize topics such as nomenclature, appearance, production regions, harvesting and post-harvest handling and medicinal processing (pao zhi). Discussions on medicinal effects primarily discuss the therapeutic effects of a medicinal on the body, while discussions of its medicinal nature primarily focus on the theoretical explanation behind these effects, such as nature and flavor, entering channels, and its ascending, descending, floating or sinking nature.

Ancient Bencao texts document the fundamental knowledge and refinement of ideas inherited from prior generations, and represents a rich resource for modern research and development. According to The complete collection of traditional texts on Chinese materia medica, edited by The Association of Chinese Culture Research, more than 800 works and 100,000 articles on Chinese medicinal materials were collected from 200 C.E. to 1911 [1]. In the Bencao texts, many contents including names, medicinal properties, indications, production areas, harvesting times, processing methods, differentiations and prescriptions were recorded. The huge literatures are important for the further study on Chinese Medicinal Materials (CMMs). The current paper outlines the contents and modern research of Bencao texts. Moreover, the impact of Bencao texts on modern research of CMMs is summarized. The purpose of current paper is to arouse the attention to Bencao texts and the intellectual property rights protection of the traditional knowledge recorded in Bencao texts.

\section{Contents of Bencao texts}

\section{Main Bencao texts in the past dynasties}

Since the end of warring states period, the Bencao texts recorded the knowledge of medicinal materials has appeared. In the excavation of Mawangdui Han Tomb, 52 Bing Fang (prescriptions for fifty-two diseases, 五十二病方) written in the warring states period was found. From the unearthed Fuyang bamboo slips of Han dynasty, Wang Wu (Everything, 萬物) also written in the warring states period was found and it records the medicinal knowledge of some materials. In many early writings, one book named as The Divine Husbandman's Classic of Materia Medica (Shen Nong Ben Cao Jing, 神農本草經) stands out. This book was compiled in the late Eastern Han Dynasty (c. 200 C.E.) which is the earliest whole materia medica. It summarizes 
medicinal experiences as of the Han Dynasty. In this book, 365 medicinals including 237 herbal substances are recorded and classified into three categories: superior, intermediate and inferior according the efficacy and toxicity. Entries for each substance include name, tastes, properties and medical applications. The other names, harvesting details, processing methods, production areas and macroscopic descriptions of some medicinal plants are also recorded briefly. This book is known only from compiled versions dating from the Ming Dynasty (c. 1400 C.E.), as the original book has been lost. Since then, this book was constantly expanded, updated and amended. Those Bencaos compiled on the basis of The Divine Husbandman's Classic of Materia Medica (Shen Nong Ben Cao Jing) are named as the main Bencaos. Figure 1 showed the development of main Bencaos. Some prominent representatives are introduced below (The Association of Chinese Culture Research, 1999):

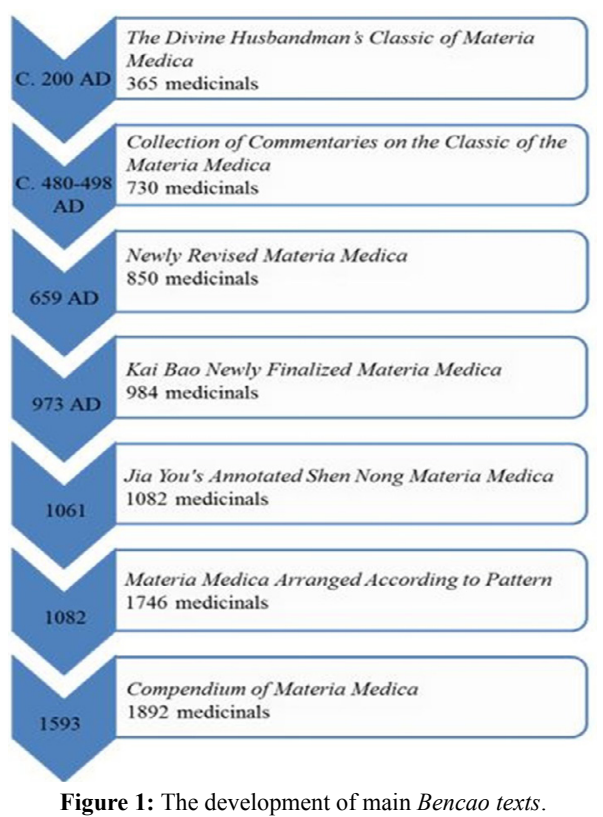

Collection of commentaries on the classic of the materia medica (Ben Cao Jing Ji Zhu, 本草經集注): It was compiled by Tao HongJing, a physician of the Liang Dynasty, by revising The Divine Husbandman's Classic of Materia Medica (Shen Nong Ben Cao Jing) and adding another 365 medicinals to increase the total to 730 medicinals. Tao distinguished the text quoted directly from The Divine Husbandman's Classic of Materia Medica (Shen Nong Ben Cao Jing) from his own annotations through the use of red and black ink. Each entry includes name, tastes, properties, toxicity or non-toxicity, medical applications, other names, production area, harvesting time, the compatibility of medicinals, etc. Figure 2 showed the record of "Shu (杧)" which the red word belonging to the content of The Divine Husbandman's Classic of Materia Medica (Shen Nong Ben Cao Jing).

Newly Revised Materia Medica (Xin Xiu Ben Cao, 新修本草): Text-based records have always made up the primary body of historical Chinese materia medica literature. However, as the range of recorded medicinals expanded, text alone was insufficient to convey information regarding the appearance of medicinals, compelling authors to add explanations and illustrations. Commissioned by the government of the Tang Dynasty in 659 C.E., this 54-volume Bencao text documenting 850 medicinals was compiled by experts according to the national survey, and was composed of three sections: written text, drawings of medicinals and captions for the illustrations. The new notes and medicinals are marked by words. In addition to being China's first illustrated materia medica, it is considered to be the world's first national pharmacopoeia. All the drawings were in full-color in the original text. Unfortunately, the original has been lost, and the only extant versions were reconstructed by later generations of editors.

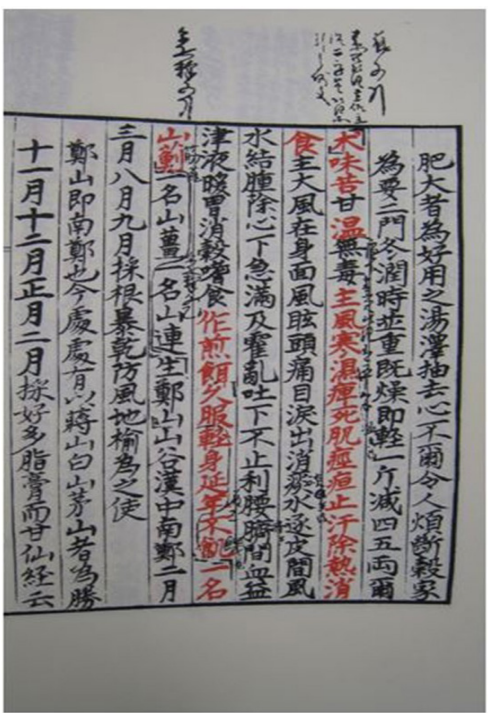

Figure 2: The record of “Shu (术)" in collection of commentaries on the classic of the materia medica which the red word belonging to the content of The Divine Husbandman's Classic of Materia Medica.

Kai Bao Newly Finalized Materia Medica (Kai Bao Ben Cao, 開 寶本草): commissioned by the government of the Song Dynasty in 973 C.E., this Bencao text took Newly Revised Materia Medica (Xin Xiu Ben Cao) as reference and involved the essence of other Bencao texts, such as Supplements to Newly Revised Materia Medica (Ben Cao Shi Yi, 本草拾遺). It records 984 medicinals and the different notes from different times including new added notes are marked by words.

After the development of the printing press in the Song Dynasty, the use of different character sizes was substituted to preserve the distinction of red vs. black ink, and practical black and white illustrations emerged. Although black and white images cannot match the clarity of full color, the comparatively low cost achieved through the use of the printing press allowed text and illustrations to become widely distributed, and the invention of the printing press greatly promoted the development of materia medica literature. Kai Bao Newly Finalized Materia Medica (Kai Bao Ben Cao) is the first printed Bencao literature.

Materia Medica of the Jiayou Era (Jia You Ben Cao, 嘉祐本草): Commissioned by the government of the Song Dynasty and edited by Zhang Yu-Xi and other famous physician, this Bencao published in 1061 C.E. records 1082 medicinals including 17 newly discovered ones. Based on Kai Bao Newly Finalized Materia Medica (Kai Bao Ben Cao), the data from many previous Bencaos were added in this book with precise and clear citation. At the same time, another officer 
Su Song was assigned for compiling Illustrated Classic of Materia Medica (Ben Cao Tu Jing, 本草圖經). It records 780 medicinals with 933 drawings. This book summarizes practical medicinal use and medicinal differentiation experience in the Song Dynasty. Su Song carried out a national survey for drawing the images of medicinals from each region and the addition of images from material medica texts from the Imperial collection. These images were made into black and white printing plates, and this publication is known as the world's first printed atlas of medicinal substances. Later generations of materia medica texts reproduced these images or their modifications extensively, and this text offers significant academic value for research into the origins of medicinal substances up through the Song Dynasty period.

Here, another famous Bencao text should be pointed out. Completed in 1505 C.E., Essentials of Materia Medica Distinctions (Ben Cao Pin Hui Jing Yao, 本草品匯精要) was a Ming Dynasty government-commissioned materia medica that contained 1815 medicinals [2]. It featured 1367 illustrations that were produced by artists selected from the imperial palace. About half of these illustrations were new, and the other half were colored and modified images based on black and white illustrations from Illustrated Classic of Materia Medica (Ben Cao Tu Jing). Many of the new images were very realistic representations of the medicinals in their natural environment. However, shortly after the book was completed, its sponsoring Emperor Xiao Zong died. In the aftermath of his death, the chief editor Liu Wen-Tai and others were charged as criminals, and the book was stored in the palace and kept out of circulation. Thus, later generations were only transmitted via hand-written copies. Therefore, some drawings of Illustrated Classic of Materia Medica (Ben Cao Tu Jing) can be traced back in Essentials of Materia Medica Distinctions (Ben Cao Pin Hui Jing Yao). Figure 3 gives three examples of the drawings.

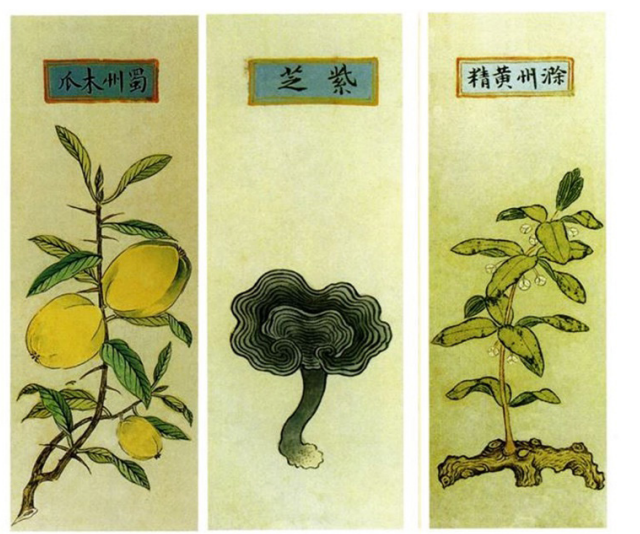

Figure 3: Illustrations from Essentials of Materia Medica Distinctions.

Materia medica Arranged According to Pattern (Jing Shi Zheng Lei Bei Ji Ben Cao, which is typically abbreviated as Zheng Lei Ben Cao, 證類本草): This book was completed by a physician named Tang Shen-Wei and published in 1108 C.E Based on the combination of Kai Bao Newly Finalized Materia Medica (Kai Bao Ben Cao) and Illustrated Classic of Materia Medica (Ben Cao Tu Jing), Tang added a great deal of information and cited them from more than 240 books. It records 1746 medicinals including their synonyms, medicinal properties, indications and production areas, harvesting times, processing methods, differentiations and prescriptions. This text has been transmitted to the modern day in three textual versions known as the "Da Guan," "Zheng He" and "Shao Xing" editions. Written by one of the most respected authors of the Song Dynasty, this text collected China's Song and pre-Song Dynasty materia medica literature into a great compendium. In addition to being the most complete specialized text dedicated to medicinals from this era, it preserved the images and text from the Materia Medica of the Jiayou Era (Jia You Ben $\mathrm{Cao}$ ) and the Illustrated Classic of Materia Medica (Ben Cao Tu Jing) for later generations.

Compendium of Materia Medica (Ben Cao Gang Mu, 本草綱目): Compiled and written by Li Shizhen, a physician of the Ming Dynasty, the Compendium of materia medica (Ben Cao Gang $\mathrm{Mu}$ ) was based on Materia Medica Arranged According to Pattern (Zheng Lei Ben Cao) and first published in 1593 C.E. It records 1,892 medicinal materials including 1,095 herbal substances. Due to the huge data, Li set up a new classification system. The medicinal materials are divided into sixteen parts with the principle of "from inferior to superior, most bases to the most noble", and then each part was classified into different categories. For example, herbs were classified into herbs growing in mountain, aromatic herbs, herbs growing in wet places, poisonous herbs, trailing herbs, herbs growing in water, herbs growing in stones, moss and lichen and weeds according to their properties, morphological characteristics and growth environments. Li consulted more than 800 books and also devoted himself to actual practice by travelling many places for making on-the-spot investigations and collecting sample specimens. Therefore, Li corrected some mistakes in previous Bencaos. Under each entry, the name explanation, commentary, argument, processing, taste and property, indications, development and formula have been documented. Figure 4 showed the record of Flos Magnoliae (Xinyi). It is no doubt that the Compendium of Materia Medic embodies the peak of the historical Chinese materia medica literature with a novel structure and rich references to the existing literature. Li Shi-Zhen's son added two scrolls of medicinal illustrations preserving 1109 images, and the lone imperfection in this beautiful text is the fact that these illustrations are relatively simple and rough. Most of the medicinal images were new, but a small portion of the images were reproduced from the Illustrated Classic of Materia Medica (Ben Cao Tu Jing).

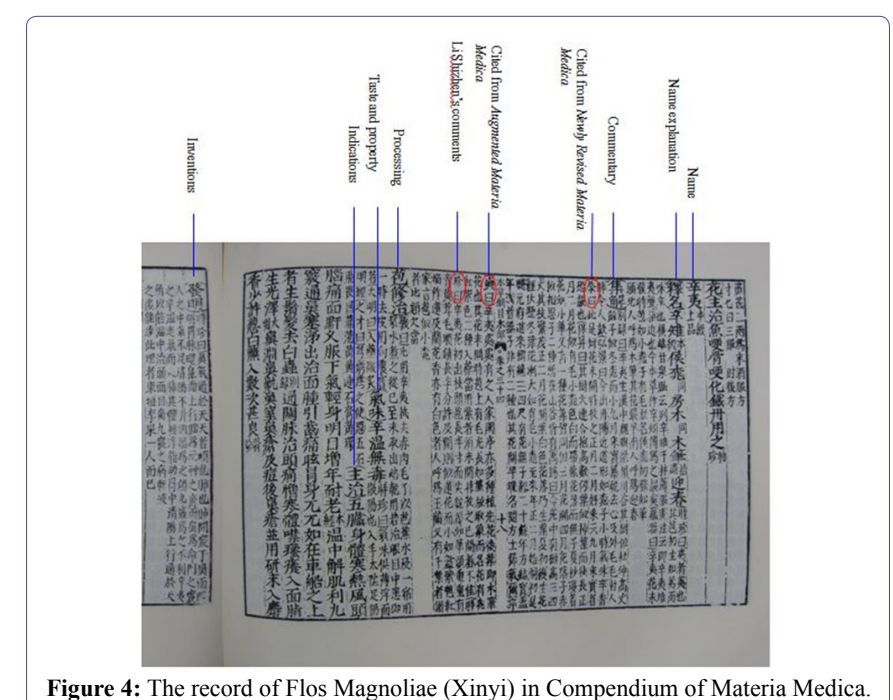

Figure 4: The record of Flos Magnoliae (Xinyi) in Compendium of Materia Medica. 


\section{Theme of Bencao texts}

The theme of Bencao texts refers to the books documented a particular topic of medicinal materials, such as processing, identification, food therapy and medicinal effects. Some prominent representatives are introduced below.

Bencaos documented processing: In our previous paper, we have mentioned the most important Bencao texts related to processing were: Grandfather Lei's Treatise on Medicinal Processing (Lei Gong Pao Zhi Lun, 雷公炮多論) written in the Northern and Southern Dynasties period (ca. 500 C.E.), Processing Methodology (Pao Zhi Da $\mathrm{Fa}$, 炮多大法) published in 1662 C.E., and Xiu Shi Guidelines for Processing (Xiu Shi Zhi Nan, 修事指南), written by Zhang Zhongyan in Qing dynasty [3]. Grandfather Lei's Treatise on Medicinal Processing is the first specialized text on medicinal processing.

In 1591 C.E., the Concise Addendum to Grandfather Lei's Treatise on Herbal Processing (Bu Yi Lei Gong Pao Zhi Bian Lan, 補遺雷公 炮製便覽) was completed. This text contains the greatest known collection of color illustrations and is the most complete, with rare and unique content. It is composed of 14 scrolls that are divided into 10 sections, covering metals/stones, herbs, woods, human, beasts, birds, worms/fish, fruits, grains and vegetables. 1193 color illustrations depict 957 medicinals; 829 illustrations came from the Essentials of Materia Medica Distinctions (Ben Cao Pin Hui Jing Yao), and 293 had never been previously seen. The color illustrations primarily show the appearance and processing of medicinals. In addition to being an important reference point as a Ming Dynasty color materia medica, this text also preserved the greatest number of illustrations from the Essentials of Materia Medica Distinctions (Ben Cao Pin Hui Jing Yao). The original text disappeared for 400 years and only recently resurfaced. It contains 219 illustrations of medicinal processing scenes that provide insight into ancient processing practices. As shown in figure 5 (illustrating steps of processing the medicinal ba dou, such as crushing, heating and pressing), this book is akin to an ancient illustrated version of standard operating procedures in Chinese medicinal processing.

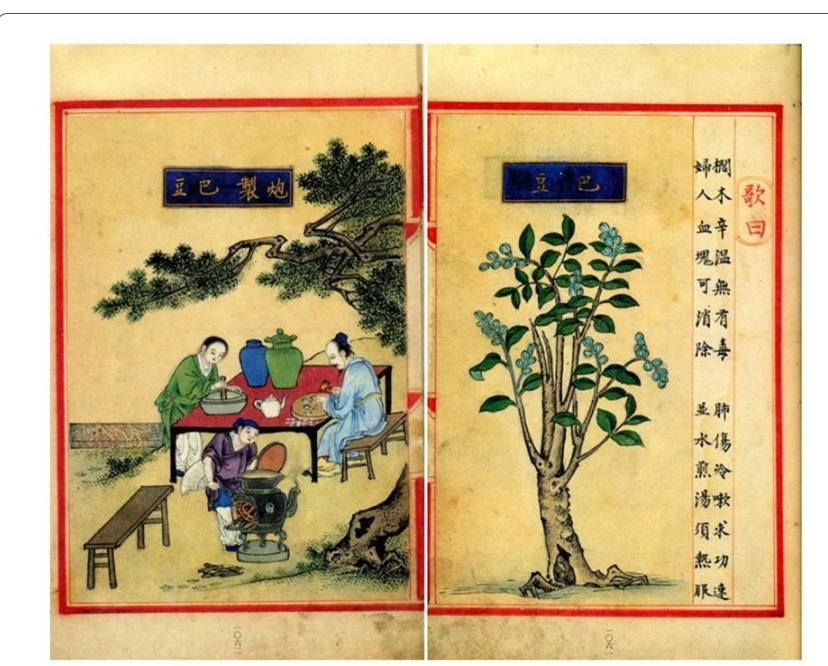

Figure 5: Steps of processing the medicinal $b a$ dou (left) and the plant drawing of $b a$ dou (right) in the Concise Addendum to Grandfather Lei's Treatise on Herbal Processing.
Bencao texts documented identification: Identification the source of medicinal materials is an important issue in Bencao texts. Since The Divine Husbandman's Classic of Materia Medica (Shen Nong Ben Cao Jing), each main Bencao text documented the identification knowledge. Specially, Li Shi-Zhen comprehensively checked and corrected the source of most of the medicinal materials recorded in the Compendium of Materia Medica (Ben Cao Gang Mu). In 1116 C.E., the text Extension of the Materia Medica (Ben Cao Yan Yi, 本 草衍義) was completed by Kou Zong-Shi. Kou had extensive personal experience and added comments about the differentiation of authentic and incorrect medicinals [4]. Later on, Kou was regarded as an authority in medicinal identification, holding a position similar to that of a modern-day expert in charge of an authentication lab. Later generations often printed and transmitted the text of his book together with the Materia Medica Arranged According to Pattern (Zheng Lei Ben Cao).

After the Song dynasty, the dispensing of medicinal materials and clinical diagnosis separated seriously and the specific monograph related to identification appeared. The Origin of Materia Medica (Ben Cao Yuan Shi, 本草原始) is a representative book which written by Li Zhong-Li in 1612 C.E. [5]. Composed of 12 scrolls covering 508 medicinals, it is considered to be the most distinctive specialized text on medicinal differentiation in the historical materia medica literature. It notably featured comparative illustrations of the medicinal material instead of the whole plant or substance, with 442 very realistic illustrations combined with text. Some medicinals had notes and annotations to clearly point out distinguishing features, and later generations of clinical texts often reproduced its illustrations of medicinal materials. Figure 6 shows the drawing of Radix Angelica sinensis (danggui). This identification knowledge is mostly from practice which provides important references for studying the kinds of medicinal materials at that time.

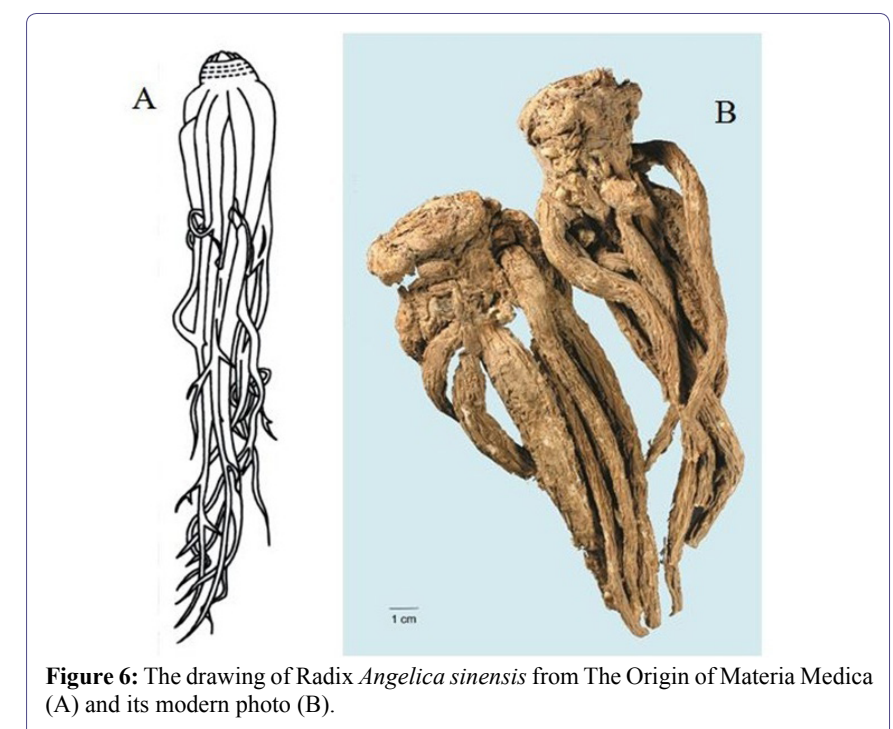

(A) and its modern photo (B).

In 1848, the Qing Dynasty botanist Wu Qi-Jun completed the Illustrated Reference of Botanical Nomenclature (Zhi Wu Ming Shi Tu Kao, 植物名實圖考). In addition to being an expert in botany, Wu was an elite scholar, earning the honor of the highest score in the traditional Chinese national civil service examination. He served 
as the governor of Hunan, Hubei and Yunnan, and he was fond of observing the plants in each region that he was posted to. He drew pictures of all the plants that he observed, and later completed the text from Henan province's Ji Gong Mountain, where he spent three years immersed in research and concentration while mourning his parents. His 38-volume book recorded 1,714 plant species, and included 1790 illustrations and nearly 1,500 sketches. The images in this text are the most accurate representations found in ancient Chinese Materia Medica literature, and this book serves a bridge linking ancient Chinese herbology to modern botany and agricultural science. Figure 7 gives two examples about the Illustrations of ren shen and sha shen.
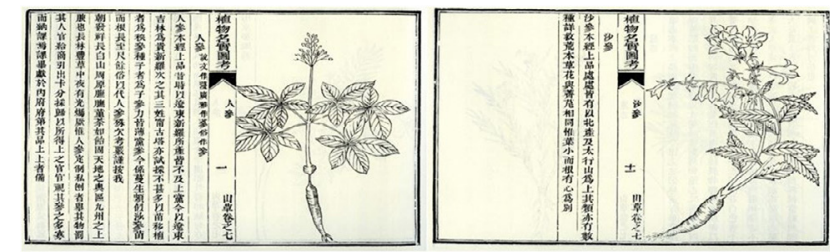

Figure 7: Illustrations of ren shen (left) and sha shen (right) from the Illustrated Reference of Botanical Nomenclature.

Bencao texts documented food therapy: This kind of book records the medicinal knowledge of food materials. In the Prescriptions worth a Thousand in Gold for Every Emergency (Bei Ji Qian Jin Yao Fang, 備急千金要方) written by Sun Si-Miao in 652 C.E., volume 26 specially introduced food therapy which is the earliest monograph about this aspect. In the Compendium of Materia Medica (Ben Cao Gang $\mathrm{Mu}$ ), it was cited as Qian Jin Shi Zhi. It records 154 food materials including their taste and property, toxic or non-toxic, effects, indications, compatibility and incompatibility. At the beginning of eight century, Zhang Ding-Zeng updated his teacher Meng Xian's book Tonic Formula (Bu Yang Fang, 補養方) and renamed it as Food Therapy Materia Medica (Shi Liao Ben Cao, 食療本草). This book specified the food therapy. Although the original book has been lost, the later people compiled 260 kinds of food from other Bencaos scattered. One of famous books about food therapy is Principles of Correct Diet (Yin Shan Zhengyao, 飲膳正要) finished in 1330 C.E. This book has distinct Mongolian features. Commissioned by the government of the Ming Dynasty in about 1503 C.E., Food Materia Medica (Shi Wu Ben Cao, 食物本草) was compiled by Imperial Academy of Medicine. It records 386 foods including the taste and property, effects, indications and usages. Drew by Court painter, 492 color drawings is attached. In Qing Dynasty, Sui Xi Ju Diet Record (Sui Xi Ju Yin Shi Pu, 隨息居 飲食譜) written by a famous physician Wang Shi-Xiong in 1861 was a representative book about food therapy. It records more than 330 common foods and discusses the effects of food tastes and properties to human body and medical use according to the theory of Traditional Chinese Medicine (TCM).

Bencao texts documented medicinal effects: This kind of texts discusses the therapeutic effects of a medicinal on the body according to the medicinal nature. Except for the identification of medicinal materials, the above mentioned Extension of the Materia Medica (Ben Cao Yan Yi) written by Kou Zong-Shi also discusses the medicinal effects. Kou applied the basic theory of The Medical Classic of the Yellow Emperor (Huang Di Nei Jing, 黃帝內經) to elucidate the medicinal effects. In Jin-Yuan dynasties, the discussion on medicinal effects blossomed. The representative book is Materia Medica for Decoctions (Tang Ye Ben Cao, 湯液本草). This book records 242 medicinal materials and summarizes the theory of medicinal effects and medicinal experiences generated in Jin-Yuan dynasties [6]. The theories from famous physicians at that time, such as Zhang Yuan-Su and Li Dong-Yuan, were cited, which reflected the medicinal effects and indications. In Ming Dynasty, a famous physician Miao Xi-Weng wrote Explanation of The Divine Husbandman's Classic of Materia Medica (Shen Nong Ben Cao Jing Shu, 神農本草經疏). He focused on the medicinal effects and indications of The Divine Husbandman's Classic of Materia Medica (Shen Nong Ben Cao Jing) and elucidated them based on the taste and property, morphological features, color and channel tropism as well as his clinical experiences [7]. In Qing Dynasty, several Bencaos related to the elucidation of the medicinal effects and indications of The Divine Husbandman's Classic of Materia Medica (Shen Nong Ben Cao Jing) were published: Reading The Divine Husbandman's Classic of Materia Medica(Shen Nong Ben Cao Jing Du, 神農本草經讀) written by Chen Xiu-Yuan, One Hundred Record of The Divine Husbandman's Classic of Materia Medica (Shen Nong Ben Cao Jing Bai Zhong Lu, 神農本草經百種錄) written by Xu Da-Chun, Explanation and Demonstration of The Divine Husbandman's Classic of Materia Medica (Ben Jing Shu Zheng, 本 經疏證) written by Zhou Shu and Speculation on Materia Medica (Ben Cao Si Bian Lu, 本草思辨錄) written by Zhou Yan. Additionally, Essential Notes of Materia Medica (Ben Cao Bei Yao, 本草備 要) written by Wang Ang, is one of the most popular book [8-12]. In the first volume, the general introduction of medicinal effects and the discussion of taste and property, channel tropism and processing were written. It records 478 medicinal materials and focuses on the effects and indications. This book is considered to be the essential book for the beginners of Traditional Chinese Medicine (TCM) at that time. Today, the status of the Essential Notes of Materia Medica (Ben Cao Bei Yao) is particularly high in Taiwan, and it is still used to train beginning TCM doctors in Taiwan.

\section{Local Bencao texts}

Local Bencao texts refer to the books on medicinal materials used in a particular area. The Descriptions of Plants in the South (Nan Fang Cao Mu Zhuang, 南方草木狀) written in 306 C.E., by Ji Hanis one of the earliest local floras in the world [13]. It mainly records the plants in Lingnan district and some imported medicinal materials. Generally, the morphology, growing conditions, taste and property, functions, production areas and related historical anecdotes were introduced.

The Lü Chan Yan Materia Medica (Lü Chan Yan Ben Cao, 履睌 岩本草) was the first illustrated materia medica dedicated to local medicinal plants, focusing on the area around modern day Hangzhou [14]. It was completed in 1220 C.E., in the Southern Song Dynasty. Copies dating back to the Ming Dynasty have been preserved, and 206 folk medicinals are referenced with beautiful color illustrations. Figure 8 gives two examples about the drawings of hu er cao and che qian cao.

The Southern Yunnan Materia Medica (Dian Nan Ben Cao, 滇南 本草) is the earliest and more completed local Bencao text, which is earlier about 100 years than Compendium of Materia Medica (Ben $\mathrm{Cao}$ Gang $\mathrm{Mu}$ ). It was written in about fourteen to fifteen century by Lan Mao, a physician of Ming Dynasty. It records the medicinal materials of Yunnan district with distinct national and local folk characteristics. The author went to the folk deeply, collected herbal medicinal materials, treated diseases for local peoples, gathered the folk medicinal experiences and spent more than twenty years for compiling this book. This book has a wide range of influence in Yunnan. 


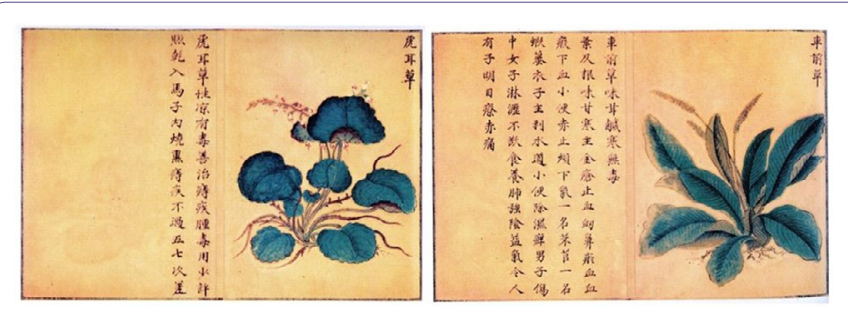

Figure 8: The drawings of hu er cao (left) and che qian cao (right) from the Materia Medica from Steep Mountainsides.

As for a particular area, many local Bencao texts relate to Bashu or Lingnan districts as the former has rich herbal resources and the latter has the habit of treating diseases by herbal materials. The main Bencaos record the medicinal materials of Bashu district include Tian Bao's Materia Medica (Tian Bao Ben Cao, 天寶本草) recorded 189 herbal medicinal materials, Nature Classification of Herbal Medicinal Materials (Fen Lei Cao Yao Xing, 分類草藥性) recorded 433 herbal medicinal materials and Convenient Formula from Herbal Medicinal Materials (Cao Mu Bian Fang, 草木便方) recorded 508 medicinal materials and about 700 formula. These books were finished in Qing Dynasty [15].

Three important Bencao texts about the herbal medicinal materials of Lingnan district include Essential Notes of the Nature of Raw Herbal Materials (Sheng Cao Yao Xing Bei Yao, 生草藥性備要) written by He Ke-Jian in 1711 C.E., seeking the Origin of Medicinal Materials (Ben Cao Qiu Yuan, 本草求原) written by Zhao Qi-Guang in 1848 and Collection Record of Lingnan Medicinal Materials (Lingnan Cai Yao Lu, 嶺南采藥錄) written by Xiao Bu-Dan in 1932. Among of them, the Collection Record of Lingnan Medicinal Materials (Lingnan Cai Yao Lu) has the most comprehensive contents and is the most widespread and influential. It systematically summarizes the medicinal experiences of Lingnan physicians since Qing Dynasty and comprehensively records 576 medicinal materials used in Lingnan district. More importantly, most of medicinal materials have not been recorded in Compendium of Materia Medica (Ben Cao Gang Mu) and still are used today in the folk of Lingnan district, such as Ilex asprella (Hook.et.Arn.) Champ., Polygonum chinense L. and Abrus cantoniensis Hance. The name, other names, plant morphology, medicinal part, taste and property, indications and usage are recorded in most of herbal medicinal materials.

\section{Other Bencao texts}

National Bencao texts refers to the books that record the medicinal experiences accumulated by a specific ethnic group, such as $\mathrm{Yu}$ Long Materia Medica (Yu Long Ben Cao, 玉龍本草) recording the medicinal experiences used by Naxi nationality and Jing Zhu Materia Medica (Jing Zhu Ben Cao, 晶珠本草) recording the medicinal experiences used by Zang nationality [16]. These Bencao texts written by specific language have their unique medicinal theories. In most of cases, the same medicinal material has its own special use.

Additionally, some ancient local chronicles also record some medicinal experiences of local plants. These also belong to a part of Bencao texts.

\section{Research on Bencao texts}

\section{Literature study}

For the literature study of Bencao texts, the collation and com- pilation are the main contents. Since most of ancient Bencaos was printed for many times in different times, the same Bencao text has different versions which spread in the world and their contents often appeared minor differences. For example, Compendium of Materia Medica (Ben Cao Gang $\mathrm{Mu}$ ), four main versions were $\mathrm{Hu}$ ChengLong version (also known as Jin Lin version), Xia Liang-Xin version (also known as Jiang Xi version), Qian Wei version and Zhang ShaoTang version [17]. The foreword, minor word contents, drawings and attached medical books among the four versions were distinct. Today, Compendium of Materia Medica (Ben Cao Gang Mu) has been printed more than 110 times on the basis of the four main versions. The representative collation versions of Compendium of Materia Medica (Ben Cao Gang Mu) were collated by Liu Heng-Ru, Liu Shan-Yong, Qian Chao-Chen and Zheng Jin-Sheng. Due to the differences in different printed versions, making a collation in different version is indispensable in the literature study.

As mentioned before, the original version of some ancient Bencao texts has lost but their contents have been cited in many other Bencao texts. Compiling the contents scattered in other Bencao texts as a book is an important work. The famous lost Bencao text is The Divine Husbandman's Classic of Materia Medica (Shen Nong Ben Cao Jing). It has nine compilation versions which were printed for twenty-six times. The first compilation of The Divine Husbandman's Classic of Materia Medica (Shen Nong Ben Cao Jing) was finished by Wang Yan in Song Dynasty. Unfortunately, this compilation was also lost and only its foreword was found in other book [18]. The nine versions were compiled by $\mathrm{Lu} \mathrm{Fu}$ (盧复), Sun Xing-Yan (孫星衍) and Sun Feng-Yi (孫馮翼), Gu Guan-Guang (顧觀光), Wang KaiYun (王闘運), Jiang Guo-Yi (姜國伊), Huang Shi (黃茞), Lin Wu Dong Xian Jiu Zhi (林屋洞仙九芝), Japanese Sen Li Zhi (森立之) and Shou Gu Wang Zhi Zhi (狩穀望之志), respectively. The contents of those compilations were from the citation of Materia Medica Arranged According to Pattern (Zheng Lei Ben Cao). In China, Prof. Shang Zhi-Jun contributes greatly in compiling the lost Bencaos, especially those before Song Dynasty. In his life, he compiled 18 lost Bencao texts including the above mentioned Collection of Commentaries on the Classic of the Materia Medica (Ben Cao Jing Ji Zhu), Newly Revised Materia Medica (Xin Xiu Ben Cao), Kai Bao Newly Finalized Materia Medica (Kai Bao Ben Cao), Jia You's Annotated Shen Nong Materia Medica (Jia You Ben Cao), Illustrated Classic of Materia Medica (Ben Cao Tu Jing), Grandfather Lei's Treatise on Medicinal Processing (Lei Gong Pao Zhi Lun) and Food Therapy Materia Medica (Shi Liao Ben Cao) [19]. It is no doubt that the ancient Bencao text style of writing in red and black for the original book and supplementary contents provides reliable basis for compiling the lost Bencao texts.

\section{Textual study}

In the past sixty years, the textual study on the species source of medicinal materials recorded in Bencao texts is the main content. As we know, the name of medicinal materials recorded in Bencao texts was in Chinese. However, in the modern science, each plant's name should have a Latin name. Moreover, due to the limited cognition at that time, the cases of different medicinal materials sharing one name, one medicinal material using different names and one medicinal material with more than one species source could be found in the Bencao texts written in different times. Therefore, to clarify the species source of medicinal materials recorded in Bencao texts is the first step for 
its modernization. The scholars combine the modern plant taxonomy, on-site market investigation and the records in different Bencao texts for clarify the species source of each medicinal material. At this aspect, Xie Zong-Wan is a distinguished scholar. He summarized the methods for clarify the species source of medicinal materials and published the related monographs "Study on species of Chinese Materia Medica" [20]. Although many works on the clarification of species source have been carried out, the species sources of many medicinal materials recorded in ancient Bencao texts are still unknown. According to our statistics, more than 300 medicinal materials recorded in Compendium of Materia Medica (Ben Cao Gang Mu) have not been clarified their species sources.

Additionally, the textual study on ancient processing experiences has been well summarized by Wang Xiao-Tao [21]. Processing is the unique issue of Chinese medicinal materials. Except for the Bencao texts documented processing, many ancient processing experiences scattered in the Main Bencao texts and medical books. These precious experiences are important for the standardization of processing procedures and probing the processing mechanism.

As the medicinal experiences in different times and locations have some differences, the production areas, medicinal parts, medicinal effects, indications as well as tastes and properties may vary. For example, Bulbus Lilii (bai he), in The Divine Husbandman's Classic of Materia Medica (Shen Nong Ben Cao Jing), its property is neutral and it can treat abdominal distension and stomach pain, and it has the effects of invigorating spleen-stomach and replenishing qi as well as facilitating excretion; today, in Chinese pharmacopoeia, its property is cold and it can treat coughs, hemoptysis, insomnia, palpitation and neurasthenia, and it has the effects of nourishing yin, moistens the lung, clearing the heart and calming the mind [22]. To clarify the changes will be helpful for the clinical application of medicinal materials.

\section{Inspiration from Bencaos on Modern Research of Chinese Medicinal Materials}

\section{Develop new drugs}

In Bencao texts, a great deal of medicinal experiences has been recorded. These traditional experiences are the valuable sources for developing new drugs. A famous example is antimalarial drug-qinghaosu (artemisinin), which was isolated from Chinese medicinal plant Artemisia annua $\mathrm{L}$. The discovery of artemisinin and its derivatives is another milestone in the history of antimalarial drug research after quinine [23]. In 2015, Chinese scientist Tu You has been awarded the Nobel Prize in medicine for her contribution to the discovery of artemisinin, a drug therapy for malaria that has saved millions of lives across the globe, especially in the developing world. Tu discovered artemisinin was inspired by a passage in the Handbook of Prescriptions for Emergencies (340 C.E., 时後備急方) by Ge Hong that referenced qingha's malaria-healing capacity. It said "take a handful of qinghao, soak in two sheng of water, strain the liquid, and drink". Therefore, it is also an exemplary case of utilization of Chinese traditional medicinal experiences.

In China, many new drugs were discovered from Chinese medicinal materials according to the traditional medicinal experiences, for examples, puerarin isolated from Pueraria lobata (Willd.) Ohwi is now being used as a vasodilator, raceanisodamine isolated from
Scopolia tangutica Maxim. Is being used as an anticholinergic, and huperzine A isolated from Huperzia serrata as a cholinesterase inhibitor. Up to now, more than twenty-five chemical drugs that originate from Chinese medicinal materials or via chemical modification of phytochemicals have been recorded in the Chinese Pharmacopeia [22].

In the "Directive 2004/24/EC of the European Parliament and of the Council of 31 March 2004 Amending, as regards Traditional Herbal Medicinal Products, Directive 2001/83/EC on the Community Code Relating to Medicinal Products for Human Use" issued by the European Parliament and the Council of the European Union (The European Parliament and the Council of the European Union, 2004) and "Guidance for Industry-Botanical Drug Products" issued by the United State Food and Drug Administration (USFDA) in June 2004, the research and development of botanical drug products have been rapidly increased. VEREGEN ${ }^{\circledR}$ (sinecatechins) Ointment is the first botanical drug approved for prescription use by USFDA [24-26]. It is made from the extract of green tea leaves. On Dec 31, 2012, the USFDA approved Fulyzaq ${ }^{\mathrm{TM}}$ (crofelemer) $125 \mathrm{mg}$ delayed-release tablets for the symptomatic relief of non-infectious diarrhea in adult patients with Human Immunodeficiency Virus (HIV) / Acquired Immune Deficiency Syndrome (AIDS) on Anti-Retroviral Therapy (ART), which is derived from a botanical source [27].

Distinctly, it has a great advantage to explore new drugs from the medicinal materials recorded in Bencaos with rich medicinal experience.

\section{Evaluate quality and safety}

In ancient times, the quality evaluation of medicinal materials depended on the evaluator's experience; distinguishing genuine or counterfeit, superior or inferior of CMM was done by morphological investigation using one's five senses, namely by observing, touching, smelling, tasting and/or testing by some other ways [28]. Historical materia medica texts contain abundant records derived from practical experience that illustrate how to differentiate the authenticity and quality of medicinals based on characteristics such as appearance, color, odor and taste. For example, Radix et Rhizoma Rhei (da huang), according to Illustrated Classic of Materia Medica (Ben Cao Tu Jing), Compendium of Materia Medica (Ben Cao Gang Mu) and The Origin of Materia Medica (Ben Cao Yuan Shi), herbal sample with conspicuous "brocaded patterns" ("brocaded patterns" refers to the appearance of woven or marbled patterns formed by the whitish parenchyma interweaving with reddish brown rays and/or anomalous vascular bundles to form reticulations observed on the outer surface and transverse section when cork is removed.) was evaluated as higher quality for medicinal use (Figure 9). The modern study has demonstrated that the secondary metabolites were mainly accumulated in brown ergastic substances of rays in normal and anomalous vascular bundles with higher contents, which supported the traditional quality authentication of Radix et Rhizoma Rhei by macroscopic characteristics [29].

In the herbal market, many specifications or grades of a certain CMM can be found. Most of the different specifications or grades of CMMs have visibly distinct morphological features. Certainly, the traditional experience recorded in Bencao texts has value and is often, appropriately, used for the quality evaluation and classification of different specifications or grades of CMMs. However, pharmaceutical quality may or may not be linked to every feature; and may be linked 
to different degrees. Sometimes, the traditional experience on quality evaluation of medicinal materials recorded in different Bencao texts is controversial; and may be different from today's experience. For example, Bupleuri Radix (chai hu), The Origin of Materia Medica (Ben Cao Yuan Shi) considers that root like a rat' tail (refers to small and long root) is of good quality; in contrast, Materia Medica of China (Zhong Hua Ben Cao) published in 1999 considers that root of a bigger size without fibrous root are superior [30]. Therefore how to validate the traditional experience by modern techniques and judge them as true or false views, with regard to pharmaceutical quality, is an important issue.

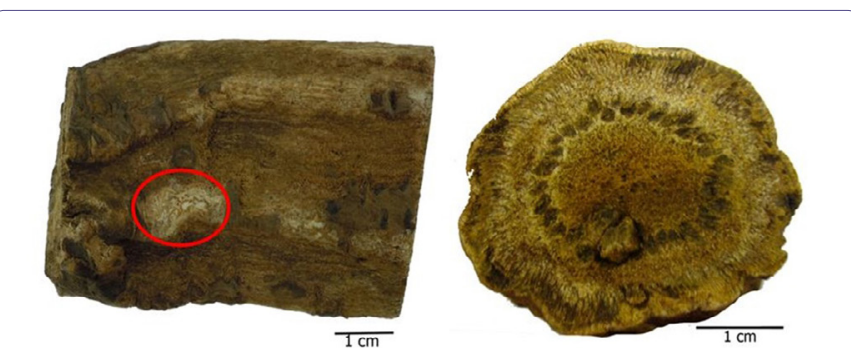

Figure 9: The conspicuous “brocaded patterns” of Radix et Rhizoma Rhei (da huang).

For the safety of Chinese medicinal materials, "Aristolochic Acid Incidents" are the distinguished cases including Aristolochiae Fangchi Radix (guang fang ji) being substituted as Stephaniae Tetrandrae Radix (fang ji) in the slimming drugs [31], Aristolochiae Manshuriensis Caulis (guan mu tong) being substituted as Akebiae Caulis (mu tong) in Longdan Xieganwan, the confusion of Herba Solani Lyrati (baiying, also named as baimaoteng) and Herba Aristolochiae Mollissimae (xungufeng, also named as bai mao teng), and the wrong use of medicinal parts of Radix et Rhizoma Asari (xixin) [32-34]. Further investigations of those cases have indicated that the accidents happened is closely related to the neglect of checking Bencao texts. According to the records of ancient Bencao texts, the information of medicinal materials including other names, morphological features of original plants, medicinal parts, medicinal effects and indications were clearly recorded. Therefore, the confused nomenclature, species sources and medicinal parts can be clarified by checking the Bencao texts. For example, in the ancient Bencao texts, the root of Herba Asari should be used as medicinal part instead of aerial or entire plant. Moreover, it also recorded that less than one "Qian" (an ancient weight units in china which was equal to $3.75 \mathrm{~g}$ ) of Radix et Rhizoma Asari was used each time in prescription, that is, the dosage of powdered root of Herba Asari should not exceed 3 gram.

From above, attaching importance to the record of Bencao texts will be helpful for modern evaluation of quality and safety of CMMs.

\section{Develop new resources}

Worldwide demands from the pharmaceutical and research industries are exerting unprecedented pressures on China's supplies of medicinal materials. At the same time, economic development and urban expansion are reducing the habitats where wild herbs grow. In other words, demand is increasing while resources are shrinking and quality is suffering as well. As mentioned above, all kinds of Bencao texts contain abundant knowledge of Chinese medicinal materials, most of which are plants. In the Chinese Pharmacopoeia, 616 medicinal materials are recorded which can be thought as the commonly used medicinal materials [22]. According to the Compendium of Materia
Medica (Ben Cao Gang Mu), 1,892 medicinal materials including 1,095 herbal substances are recorded. More resources should be developed from the Bencao texts. The modern studies including chemical analysis and pharmacological evaluation should be carried out on the rarely used medicinal materials recorded in Bencao texts.

Besides, comparing the data in these ancient Bencao texts reveals the changes in source species of medicinal materials over different historical periods. For example, the same name of a medicinal material with different source plants can be found in the records of different Bencao texts. Systematic textual investigation of Bencao texts written in different time can lead to the discovery of new source species of medicinal materials. For example, according to the different Bencao texts, more than one source plants of Flos Magnoliae including one with white flower and one with reddish-purple flower were recorded. Based on the records of production areas and morphological features in Bencao texts, a survey of resources and species was carried out. It was found that the species of Magnolia biondii Pamp. Was the main source plant of Flos Magnoliae. At the same time, a new species named as Magnolia pilocarpa ZZ Zhao et al., ZW Xie was found $[35,36]$. Moreover, to clarify the unknown species sources of many medicinal materials recorded in ancient Bencao texts is also helpful for the development of new resources.

\section{Conclusion}

Bencao texts record a lot of knowledge of CMMs including numerous formulas which belong to the traditional wisdoms accumulated by the ancient people's experience. Except for focusing on the study of Bencao texts themselves, great efforts are needed to validate scientifically those traditional wisdoms. Moreover, Bencao texts could provide initial guidance for developing new drugs to benefit health care.

On the other hand, with the rapid development of the research on CMMs, more and more attention has been paid on the Bencao texts with their great economic value and cultural value by both the domestic and international industries and academic fields. Today protecting the intellectual property of traditional knowledge from pharmaceutical exploitation is a significant issue. There have been examples of drug companies exploiting the knowledge of traditional healers, resulting in profitable drugs that do not benefit the traditional herbalists that "discovered" them. In India, the government has created the Traditional Knowledge Digital Library (TKDL) for codifying traditional knowledge existing in local languages on Indian systems of medicine since 2001. The aim is to prevent the misappropriation of traditional knowledge at international patent offices, as well as to derive cues for bioprospection. In 2003, the India government established The National Biodiversity Authority (NBA). Any individuals or entities require the approval of the NBA when they use biological resources and associated knowledge occurring in India for commercial or research purposes or for the purposes of bio-survey or bio-utilisation. It is true that every people or entity should respect the intellectual property rights created by the local people. At this aspect, the intellectual property rights protection of Chinese medicine was weak.

In China, the knowledge related to Chinese medicine has applied the Intangible Cultural Heritage of the World for better protection. In the Bencao texts, many ancient Chinese medicine formulas have been recorded and validated by the clinic. Although these formulas have been opened, their intellectual property rights protection should be paid for attention and the researcher can apply the patents on the basis 
of optimizing the ration of each medicinal material in the formulas, probing their mechanism and medicinal substances. For the intellectual property rights protection of Chinese medicine, to establish the suitable system according to the Chinese medicine characteristics is an important work.

\section{Acknowledgement}

We are grateful to Dr. Eric Brand for his valuable comments on this paper. The study was supported by Hong Kong General Research Fund (Project ID: 12102415) and National Natural Science Foundation of China ((Project ID: 11475248).

\section{References}

1. Chinese Culture Research Association (1999) The Complete Collection of Traditional Texts on Chinese Materia Medica. Huaxia Publishing House, Beijing, China.

2. Cao H (2004) Essentials of Materia Medica Distinctions (Ben Cao Pin Hui Jing Yao). Cathay Press, Beijing, China.

3. Zhao Z, Liang Z, Chan K, Lu G, Lee EL, et al. (2010) A unique issue in the standardization of Chinese Materia Medica: processing. Planta Med 76: 1975-1986.

4. Zongshi K (2017) Augmented Materia Medica. China Medical Science Press, Beijing, China.

5. Li ZL (2007) The Origins of the Materia Medica (Ben Cao Yuan Shi). People's Medical Publishing House, Beijing, China.

6. Wang HG (2008) Materia Medica for ecoctions (TangYe Ben Cao). China Medical Science Press, Beijing, China.

7. Miao XW (2013) Explanation of The Divine Husbandman's Classic of Materia Medica (Shen Nong Ben Cao Jing Shu). Shanxi Science and Technology Press, Taiyuan, China.

8. Chen NZ (2011) Reading The Divine Husbandman's Classic of Materia Medica (Shen Nong Ben Cao Jing Du). China Medical Science Press, Beijing, China.

9. Xu DC (2011) One Hundred Record of The Divine Husbandman's Classic of Materia Medica (Shen Nong Ben Cao Jing Bai Zhong Lu). China Medical Science Press, Beijing, China.

10. Zhou S (2013) Explanation and Demonstration of The Divine Husbandman's Classic of Materia Medica (Ben Jing Shu Zheng). China Medical Science Press, Beijing, China.

11. Zhou Y (2013) Speculation on Materia Medica (Ben Cao Si Bian Lu). China Medical Science Press, Beijing, China.

12. Wang A (2005) Essential Notes of Materia Medica (Ben Cao Bei Yao). People's Medical Publishing House, Beijing, China.

13. Xi H (2009) Descriptions of Plants in the South (Nan Fang Cao Mu Zhuang). Guangdong Science and Technology Press, Guangzhou, China.

14. Zheng JS (2007) Three Precious Bencaos of South Song Dynasty. People's Medical Publishing House, Beijing, China.

15. Xie ZW, Wu JL (2001) The New Edition of Tian Bao's Materia Medica. Publishing House of Ancient Chinese Medical Books, Beijing, China.

16. Yunnan Provincial Health Department Institute for drug control (1959) Yu Long Materia Medica (Yu Long Ben Cao). Yunnan People's Publishing House, Kunming, China.

17. Ma JX, Hu NC (1984) Brief record on the carvings of Compendium of Materia Medica. Journal of Traditional Medicine 8: 57-59.
18. Shang ZJ (1982) All compiled versions of The Divine Husbandman's Classic of Materia Medica from Materia Medica Arranged According to Pattern. Jiangsu Journal of Traditional Medicine 2: 38-39.

19. Tao GS, Ni XG, Zhao HZ, Ren H (2008) Overview of Professor Shang Zhijun's researches on Materia Medica literature. Acta Universitatis Traditionis Medicalis Sinensis Pharmacologiaeque Shanghai 22: 4-8.

20. Xie ZW (1992) Studies on species of Chinese Materia Medica. Shanghai Science and Technology Press, Shanghai, China.

21. Wang XT (1998) The data collection of Chinese Materia Medica processing in the past dynasties (Ancient part). Jiangxi Science and Technology Press, Nanchang, China.

22. Chinese Pharmacopoeia Commission (2015) Pharmacopoeia of the People's Republic of China. Chinese Pharmacopoeia Commission, Beijing, China.

23. Wu Y (2009) Qinghaosu (Artemisinin)-revelations from history and reality. Progress in Chemistry -Beijing 11: 2365-2371.

24. The European Parliament and the Council of the European Union (2004) Directive 2004/24/EC of the European Parliament and of the Council of 31 March 2004 amending, as regards traditional herbal medicinal products, Directive 2001/83/EC on the Community code relating to medicinal products for human use. The European Parliament and the Council of the European Union 136: 85-90.

25. Center for Drug Evaluation and Research (2016) Botanical Drug Development Guidance for Industry. US Food and Drug Administration, Silver Spring, USA.

26. American Botanical Council (2007) FDA approves special green tea extract as a new topical drug for genital warts. American Botanical Council, Austin, USA.

27. US Food and Drug Administration (2012) FDA approves first anti-diarrheal drug for HIV/AIDS patients. US Food and Drug Administration, Silver Spring, USA.

28. Zhao Z, Liang Z, Ping G (2011) Macroscopic identification of Chinese medicinal materials: traditional experiences and modern understanding. J Ethnopharmacol 134: 556-564.

29. Liang Z, Sham T, Yang G, Yi L, Chen H, et al. (2013) Profiling of secondary metabolites in tissues from Rheum palmatum L. using laser microdissection and liquid chromatography mass spectrometry. Anal Bioanal Chem 405: 4199-4212.

30. The Editorial Committee (1999) Materia Medica of China. Shanghai Science and Technology Publishing House, Shanghai, China.

31. Stengel B, Jones E (1998) [End-stage renal insufficiency associated with Chinese herbal consumption in France]. Nephrologie 19: 15-20.

32. Martena MJ, van der Wielen JC, van de Laak LF, Konings EJ, de Groot $\mathrm{HN}$, et al. (2007) Enforcement of the ban on Aristolochic acids in Chinese traditional herbal preparations on the Dutch market. Anal Bioanal Chem 389: 263-275.

33. Liang ZT, Jiang ZH, Leung KSY, Chan CL, Zhao ZZ (2006) Authentication and differentiation of two easily confusable Chinese Materia Medica: Herbal Solani Lyrati and Herba Aristolochiae Millissimae. Journal of Food and Drug Analysis 14: 36-43.

34. Zhao ZZ, Liang ZT, Jiang ZH, Leung KS, Chan CL, et al. (2008) Comparative study on the aristolochic acid I content of Herba Asarifor safe use. Phytomedicine 15: 741-748.

35. Zhao ZZ, Xie ZW, Shen J (1987) [A new species and a variety's new nomenclature of the medicinal xin-yi (Flos Magnoliae)]. Yao Xue Xue Bao 22: 777-780.

36. Zhongzhen Z, Zongwan X, Jie S (1988) Original botanical investigation and identification of Flos Magnoliae. Institute of Chinese Materia Medica, China Academy of TCM, Beijing, China. 